The LISA Pathfinder mission

This article has been downloaded from IOPscience. Please scroll down to see the full text article.

2012 Class. Quantum Grav. 29124014

(http://iopscience.iop.org/0264-9381/29/12/124014)

View the table of contents for this issue, or go to the journal homepage for more

Download details:

IP Address: 194.94.224.254

The article was downloaded on 05/06/2012 at 09:09

Please note that terms and conditions apply. 


\title{
The LISA Pathfinder mission
}

\author{
F Antonucci ${ }^{1}$, M Armano $^{2}$, H Audley $^{3}$, G Auger $^{4}$, M Benedetti $^{5}$,

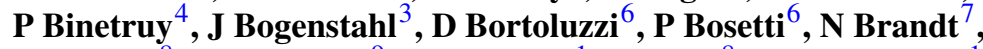 \\ M Caleno $^{8}$, P Cañizares $^{9}$, A Cavalleri $^{1}$, M Cesa $^{8}$, M Chmeissani $^{10}$,

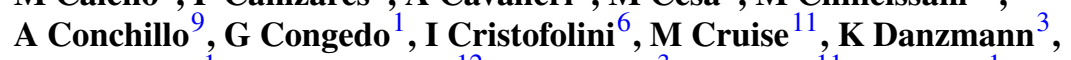 \\ F De Marchi ${ }^{1}$, M Diaz-Aguilo ${ }^{12}$, I Diepholz ${ }^{3}$, G Dixon ${ }^{11}$, R Dolesi ${ }^{1}$, \\ N Dunbar ${ }^{13}$, J Fauste ${ }^{2}$, L Ferraioli ${ }^{4}$, V Ferrone ${ }^{1}$, W Fichter $^{14}$, \\ E Fitzsimons $^{15}$, M Freschi ${ }^{2}$, A García Marin ${ }^{3}$, C García Marirrodriga ${ }^{8}$, \\ R Gerndt $^{7}$, L Gesa ${ }^{9}$, F Gilbert ${ }^{9}$, D Giardini ${ }^{16}$, C Grimani ${ }^{17}$, \\ A Grynagier $^{14}$, B Guillaume ${ }^{8}$, F Guzmán ${ }^{3}$, I Harrison ${ }^{18}$, G Heinzel $^{3}$, \\ V Hernández ${ }^{9}$, M Hewitson ${ }^{3}$, D Hollington ${ }^{19}$, J Hough ${ }^{15}$, D Hoyland ${ }^{11}$, \\ M Hueller $^{1}$, J Huesler ${ }^{8}$, O Jennrich ${ }^{8}$, P Jetzer ${ }^{20}$, B Johlander ${ }^{8}$, \\ N Karnesis ${ }^{9}$, C Killow ${ }^{15}$, X Llamas ${ }^{21}$, I Lloro ${ }^{9}$, A Lobo ${ }^{9}$, \\ R Maarschalkerweerd ${ }^{18}, \mathbf{S} \operatorname{Madden}^{8}$, D Mance ${ }^{16}$, I Mateos ${ }^{9}$,

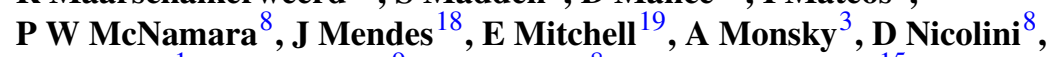 \\ D Nicolodi ${ }^{1}$, M Nofrarias ${ }^{9}, \mathbf{F}$ Pedersen $^{8}$, M Perreur-Lloyd $^{15}$, E Plagnol $^{4}$, \\ P Prat ${ }^{4}$, G D Racca ${ }^{8}$, J Ramos-Castro ${ }^{22}$, J Reiche ${ }^{3}$, J A Romera Perez ${ }^{8}$, \\ D Robertson ${ }^{15}$, H Rozemeijer ${ }^{8}$, J Sanjuan ${ }^{23}$, A Schleicher ${ }^{7}$, \\ M Schulte $^{19}$, D Shaul ${ }^{19}$, L Stagnaro ${ }^{8}$, S Strandmoe ${ }^{8}$, F Steier $^{3}$, \\ T J Sumner $^{19}$, A Taylor ${ }^{15}$, D Texier ${ }^{2}$, C Trenkel $^{13}$, H-B Tu ${ }^{1}$, S Vitale ${ }^{1}$, \\ G Wanner ${ }^{3}$, H Ward ${ }^{15}$, $\mathbf{S}$ Waschke ${ }^{19}$, P Wass $^{19}$, W J Weber ${ }^{1}$, T Ziegler $^{7}$ \\ and $P$ Zweifel $^{16}$

\footnotetext{
${ }^{1}$ Dipartimento di Fisica, Università di Trento and INFN, Gruppo Collegato di Trento, 38123 Povo, Trento, Italy

2 European Space Astronomy Centre, European Space Agency, Villanueva de la Cañada, 28692 Madrid, Spain

${ }^{3}$ Albert-Einstein-Institut, Max-Planck-Institut für Gravitationsphysik und Universität Hannover, 30167 Hannover, Germany

${ }^{4}$ APC UMR7164, Université Paris Diderot, Paris, France

5 Dipartimento di Ingegneria dei Materiali e Tecnologie Industriali, Università di Trento and INFN, Gruppo Collegato di Trento, Mesiano, Trento, Italy

${ }^{6}$ Dipartimento di Ingegneria Meccanica e Strutturale, Università di Trento and INFN, Gruppo

Collegato di Trento, Mesiano, Trento, Italy

${ }^{7}$ Astrium GmbH, Claude-Dornier-Strasse, 88090 Immenstaad, Germany

${ }^{8}$ European Space Technology Centre, European Space Agency, Keplerlaan 1, 2200 AG

Noordwijk, The Netherlands

${ }^{9}$ ICE-CSIC/IEEC, Facultat de Ciències, E-08193 Bellaterra, Barcelona, Spain

${ }^{10}$ IFAE, Universitat Autònoma de Barcelona, E-08193 Bellaterra, Barcelona, Spain

11 Department of Physics and Astronomy, University of Birmingham, Birmingham, UK

12 UPC/IEEC, EPSC, Esteve Terrades 5, E-08860 Castelldefels, Barcelona, Spain

13 Astrium Ltd, Gunnels Wood Road, Stevenage, Hertfordshire SG1 2AS, UK

15 Department of Physics and Astronomy, University of Glasgow, Glasgow, UK

${ }^{16}$ Institut für Geophysik, ETH Zürich, Sonneggstrasse 5, CH-8092 Zürich, Switzerland

${ }^{17}$ Istituto di Fisica, Università degli Studi di Urbino/INFN Urbino (PU), Italy

18 European Space Operations Centre, European Space Agency, 64293 Darmstadt, Germany

19 The Blackett Laboratory, Imperial College, London, UK
} \\ ${ }^{14}$ Institut für Flugmechanik und Flugregelung, 70569 Stuttgart, Germany
}


${ }^{20}$ Institut für Theoretische Physik, Universität Zürich, Winterthurerstrasse 190, CH-8057 Zürich, Switzerland

${ }^{21}$ NTE-SENER, Can Malé, E-08186, Lliçà d'Amunt, Barcelona, Spain

${ }^{22}$ Universitat Politècnica de Catalunya, Enginyeria Electrònica, Jordi Girona 1-3, 08034

Barcelona, Spain

${ }^{23}$ Department of Physics, University of Florida, Gainesville, FL 32611-8440, USA

E-mail: martin.hewitson@aei.mpg.de

Received 14 November 2011, in final form 26 December 2011

Published 1 June 2012

Online at stacks.iop.org/CQG/29/124014

\begin{abstract}
In this paper, we describe the current status of the LISA Pathfinder mission, a precursor mission aimed at demonstrating key technologies for future spacebased gravitational wave detectors, like LISA. Since much of the flight hardware has already been constructed and tested, we will show that performance measurements and analysis of these flight components lead to an expected performance of the LISA Pathfinder which is a significant improvement over the mission requirements, and which actually reaches the LISA requirements over the entire LISA Pathfinder measurement band.
\end{abstract}

PACS numbers: $95.55 . \mathrm{Ym}, 04.80 . \mathrm{Nn}$

(Some figures may appear in colour only in the online journal)

\title{
1. Introduction
}

The detection and observation of gravitational waves at frequencies below $1 \mathrm{~Hz}$ using laser interferometry presents challenges which can only be practically solved by moving the detector into space. The LISA mission [1] was conceived to establish a multi-link laser interferometer in space with an arm length of 5 million kilometres. The full detector comprises three satellites, each containing two test masses in free fall. The distance between each of the three pairs of test masses is measured using laser interferometry. By a suitable combination of the resulting measured phase shifts, the gravitational strain impinging on the constellation can be determined. The mission is designed to be sensitive to strains in spacetime at a level of about 1 part in $10^{20}$ at frequencies from 0.1 to $100 \mathrm{mHz}$.

The technological challenges in building an instrument such as LISA are significant and in many cases impossible to test fully on ground. As such, a technology-demonstrator mission called the LISA Pathfinder (LPF) was conceived to test some of the key technologies and to demonstrate the ability to place test masses in free fall at the level required to attain the scientific goals of a LISA-like mission. To make the Pathfinder mission achievable on a reasonable time scale and within the available cost envelope, the requirements were relaxed a little from the LISA requirements, resulting in a required free-fall purity of $3 \times$ $10^{-14} \mathrm{~m} \mathrm{~s}^{-2} \mathrm{~Hz}^{-1 / 2}$ at $1 \mathrm{mHz}$. A full description of the goals for the LPF is given in [2].

Some of the technologies to be tested are: the drag-free and attitude control system (DFACS) [3] needed to control the spacecraft in a way that leaves the free-falling test mass undisturbed at the required level; the laser interferometry, which needs to be stable and 
accurate enough to measure the position of the free-falling test mass at the picometre level; the spacecraft thrusters which need to be able to produce micro-Newton levels of thrust in the control bandwidth; and the inertial sensor system which must provide an environment for the test mass in which the test-mass position and attitude can be sensed and actuated in such a way that the free fall is not disturbed along the sensitive $(x)$ measurement axis.

In actuality, as with the LISA, a differential measurement is made between two test masses, but in the LPF they are placed about $30 \mathrm{~cm}$ apart. The first test mass is in free fall along the $x$-axis; the second test mass is electrostatically suspended at low frequencies (with a unity gain frequency around $1 \mathrm{mHz}$ ) and serves as a reference from which to measure the motion of the free-falling test mass. The overall top-level scientific requirement for the mission is expressed as a differential acceleration between the two test masses over the frequency band from 1 to $30 \mathrm{mHz}$ and is

$$
\Delta a \leqslant 3 \times 10^{-14}\left[1+\left(\frac{f}{3 \mathrm{mHz}}\right)^{2}\right] \mathrm{ms}^{-2} \mathrm{~Hz}^{-1 / 2} .
$$

The corresponding requirement on the sensitivity of the interferometer which measures the differential position of the two test masses is

$$
\Delta x \leqslant 9 \times 10^{-12}\left[1+\left(\frac{3 \mathrm{mHz}}{f}\right)^{2}\right] \mathrm{m} \mathrm{Hz}^{-1 / 2} .
$$

Although the demonstration of the technologies meeting the desired requirements is a key focus of the mission, it is only part of the overall goal. The second part is to develop a physical model of the system which is sufficiently detailed to allow a projection of the technologies and the full system performance to a LISA-like configuration.

The optimization, characterization and modelling of the satellite in flight is the main goal of the mission operations phase. There, a series of investigations will be carried out, each of which targets a particular aspect of the system, e.g., estimation of the elastic coupling (stiffness) parameters between the test masses and spacecraft, calibration of the actuators, and a full assessment of the contributing noise sources which limit the purity of the free fall.

\section{The LPF mission}

The LPF mission comprises two payloads: the LISA technology package (LTP) provided by European Member States and ESA, and the disturbance reduction system (DRS) [4] provided by NASA. Unlike those of typical scientific satellite missions, both of these payloads are tightly integrated into the spacecraft. In fact, during scientific operations, the spacecraft and payload(s) operate as one unit.

\subsection{Payload and spacecraft status}

The two payloads (the DRS and LTP) are integrated into a single spacecraft which will be launched on a dedicated launch vehicle (the current baseline is a VEGA launcher). An expendable propulsion module will be used to execute a series of apogee-raising manoeuvres before injecting the spacecraft into a transfer orbit towards the first Sun-Earth Lagrange point (L1). Just before arrival at L1, the propulsion module will separate from the spacecraft and the micro-Newton thrusters will be used to de-spin and stabilize the spacecraft, placing it in a $500000 \mathrm{~km} \times 800000 \mathrm{~km}$ Lissajous orbit around L1.

The LTP includes two cubic test masses made up of an alloy of platinum and gold. The test masses are gold coated and weigh around $2 \mathrm{~kg}$. They are housed inside enclosures made 
up of molybdenum with gold-coated sapphire electrodes mounted on the internal faces of the enclosure. These electrodes are used to read out the position of a test mass in six degrees of freedom as well as to apply forces to the test mass. The test-mass housings are located in vacuum enclosures which are mounted either side of the optical bench which forms the core of the Optical Metrology System (OMS). The test masses, housings, vacuum enclosures and associated electronics form the Inertial Sensor Subsystem (ISS), further details of which can be found in [5].

Comprised of four interferometers, a laser and modulation optics, a phasemeter and a payload computer, the OMS provides a sensitive readout of six degrees of freedom of the entire system: the position of one test mass relative to the spacecraft along an axis (the $x$-axis) joining the two test masses; the differential position of the two test masses along the $x$-axis; and the angle of each test mass around the $y$ - and $z$-axes (measured using differential wavefront sensing). Further details of the OMS are given in [6].

Most of the flight components of the LTP have been integrated onto the spacecraft and are currently undergoing, or have recently undergone, ground testing. The integration and delivery of the LTP is awaiting the completion of a suitable launch lock for the test masses. The LTP as a whole is discussed in more detail in [7].

The micro-propulsion system (MPS) is formally a part of the SC. The baseline system for the LPF is based on field-emission electric propulsion (FEEP) technology. Due to various delays in the development of the FEEP thrusters, a number of alternative systems have recently been studied by ESA. These are currently undergoing review and a selection is expected to be made around the end of 2011.

At the time of writing, there are various test campaigns underway. The SC is undergoing environmental testing, including thermal vacuum tests and integrated system testing. The OMS was recently put through a first set of flight-model performance and functionality tests, with further tests planned as the final flight optical bench and photodiodes are integrated with the rest of the OMS.

In early 2012, the SC will be put into storage to await the delivery of the LTP. Given the current schedule, the LPF is expected to launch in mid-2014.

\subsection{The experiment master plan}

The scientific return of the mission is maximized by a careful planning of the experiments to be done on orbit. These experiments aim to fulfil the two main mission goals: to achieve the purest level of free fall of the test masses and to build up a detailed physical model of the residual disturbances that limit free-fall purity. The content of the experiment master plan has been defined by the Science Working Team over a period of many years. These detailed experiment descriptions are then distilled down into discrete investigations which can be performed on the satellite. Once the investigations of a particular experiment have been identified and defined in terms of tele-command sequences, they must be tested and verified on ground prior to launch. This is done using the mission simulator which can run actual tele-command sequences and return realistic telemetry which can then be analysed.

The science phase of the mission operations is limited to 90 days for the LTP. In these 90 days, the mission timeline must be packed with pre-planned experiments and investigations. The arrangement of the investigations depends on many things, like the importance of the investigation, the state of the system before and after an investigation, and the investigation duration. Although the mission timeline will be defined before launch, it must remain flexible to changes in the sequencing of the investigations, since the actual results of the investigations could require that subsequent investigations be reordered. 


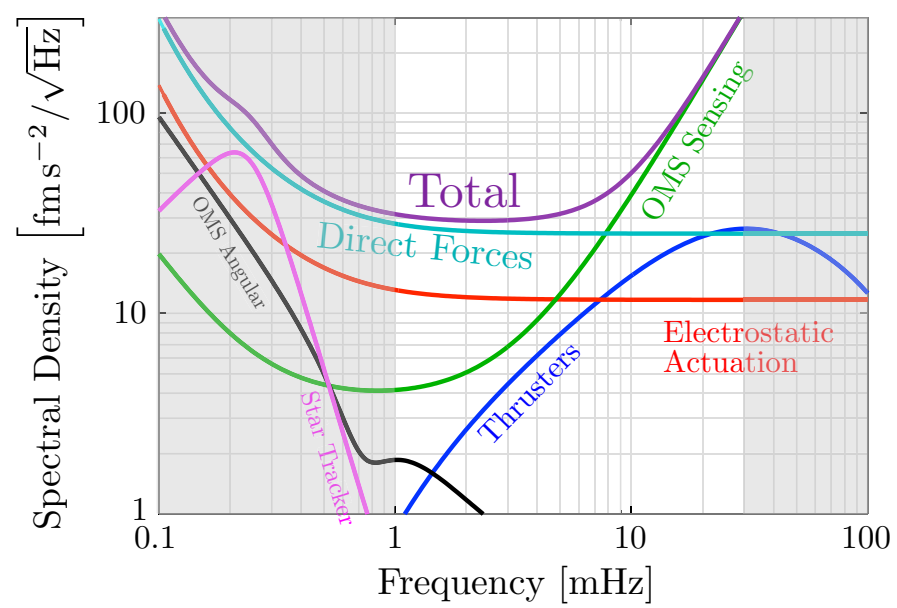

Figure 1. The differential acceleration noise budget arising from the requirements placed on the individual subsystems and noise sources. The grey areas are out of the measurement band for the LPF but are of interest for LISA.

\subsection{Data analysis}

Running the investigations back-to-back whilst maintaining some degree of flexibility in their ordering will be the key to maximizing the scientific return given the short period of scientific operations. In order to do this, the data (telemetry) from each investigation must be analysed as soon as it is received (typically within hours of the investigation being executed on-board) so that subsequent investigations can be reordered or adjusted as necessary. Given the criticality of the decisions made based on the analysis results, a flexible and robust data analysis toolbox was developed for the mission. Implemented as a MATLAB toolbox, the LISA Technology Package Data Analysis (LTPDA) toolbox [8] provides an object-oriented data analysis environment within MATLAB. It comes equipped with a large number of standard data analysis algorithms, typical for use in instrument characterization analyses, as well as a large number of higher level routines targeted for the particular investigations of the LPF. Further details about the data analysis for the LPF can be found in [9].

\section{Projected system performance}

During the mission definition phase, requirements were placed on the various subsystems so that the target performance of the system as a whole could be reached. In order to allow the direct forces acting on the test mass to be probed, other contributing noise sources-such as the sensing and displacement noise of interferometers, and the force noise of the electrostatic actuators and micro-Newton thrusters-must be kept below a certain level. Figure 1 shows the differential force noise budget assuming the subsystem requirements, indicating the main contributing groups of noise. The figure shows that the direct force noise acting on the test masses is given a dominant noise allocation in the low frequencies of the measurement band. Towards higher frequencies, the sensing noise of the OMS takes over to limit the performance. 


\subsection{Improving the projected performance}

Improvements in the projected performance of the system can arise from different sources. The main one comes from the fact that the requirements are set before construction of the flight hardware has begun and are, as such, necessarily conservative. Once the hardware is built and tested in realistic settings, we obtain more reliable estimates of the performance of individual subsystems. In addition, a detailed understanding of the differences in the environment under which the subsystems are tested compared to the environment expected in orbit could lead to further improvements. In addition to the testing and analysis of flight hardware, studies of the system as a whole can lead to data analysis methods and system-identification techniques (see, e.g., $[10,11])$, which will allow the system to be optimized in orbit, resulting in reductions of the coupling of some noise sources to differential force on the test masses. Some examples of these improvements are given below.

3.1.1. Measured performance of the electrostatic actuators. The electrostatic actuation is used to control the position of TM2 to ensure that it tracks the free-falling TM1. When estimating the differential acceleration of the two test masses, this commanded actuation is removed (see [12]). As such, any fluctuations in the applied actuation force which are not present in the commanded actuation will not be removed and will be a direct contributor to the estimated differential acceleration. These fluctuations ultimately arise in fluctuations of the ac voltages applied to produce a dc force that counteracts any gravitational imbalance within the SC. The flight models of the electronics which produce the ac voltages have now undergone direct measurement and have shown that the fluctuations are at the level of $3-8 \mathrm{ppm} \mathrm{Hz}^{-1 / 2}$ at $1 \mathrm{mHz}$. Together with detailed modelling of the expected gravitational imbalances (estimated to be around $0.65 \mathrm{~nm} \mathrm{~s}^{-2}$ ), this performance led to a lower estimate for the expected force noise fluctuations. Further discussion on this can be found in [13]. Figure 2(a) shows (solid line) the expected performance coming from these measurements together with the requirements performance. In the 1-30 $\mathrm{mHz}$ band, a considerable improvement can be seen.

3.1.2. System optimization to reduce star tracker contribution. Analysis of the entire control system has revealed that the coupling of noise from the star tracker with the differential longitudinal measurement has a dominating effect below $1 \mathrm{mHz}$. The star tracker is used to ensure that the SC does not roll about the SC-Sun axis. The signals from the star tracker are used to determine an angular error signal of the $\mathrm{SC}$ which is then fedback via suitable control functions to the drag-free coordinates of the two test masses, which results in the SC rotating to keep the two test masses centred. Through cross-couplings in the actuators, the (noisy) star tracker signals can leak into the sensitive $x$-axis measurements. Although this is out of the LPF measurement band, it is still of interest for LISA to be able to measure the best level of free fall below $1 \mathrm{mHz}$. It would therefore be advantageous to lower the contribution of the star tracker. Since the star tracker flight unit is in place already, the only way to reduce the contribution is to reduce the coupling. This can be achieved by a redesign of some of the attitude control laws. Figure $2(b)$ shows the result of one possible redesign that leads to a reduction of a factor 10 at the interesting frequencies.

3.1.3. OMS performance in the laboratory environment. The OMS has undergone engineering-model level tests and flight-model level tests. These tests, together with routine measurements of a bread-board system, show that the high-frequency sensitivity of the system should be around $6 \mathrm{pm} \mathrm{Hz}^{-1 / 2}$ compared to the requirement of $9 \mathrm{pm} \mathrm{Hz}^{-1 / 2}$. In addition, experiments on the bread-board system in the lab have shown that the low-frequency noise 


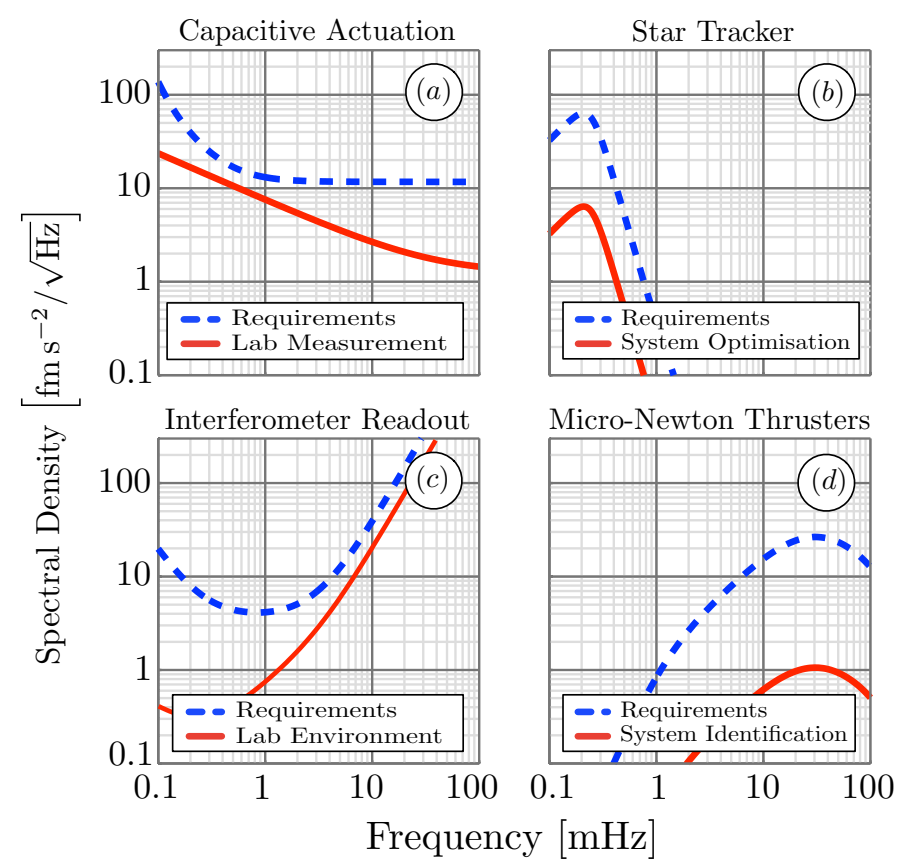

Figure 2. The different panels show the contributions of different noise sources to the differential acceleration of the two test masses. In all panels, the expected performance is contrasted against the required subsystem performance. (a) The expected performance of the electrostatic actuation subsystem after measurements of the flight units. (b) A possible improvement in the coupling of star tracker noise after a redesign of some of the attitude control loops. (c) The expected performance of the OMS based on typical performance measurements of the bread-board system as well as the performance of the engineering and flight models. This also includes an improvement at lower frequencies where the dominating effect comes from the temperature stability of the lab environment, which is expected to be much more stable in flight. $(d)$ The expected contribution of the micro-Newton thrusters when the stiffness coupling of the two TMs to the SC is matched to 1 part in 1000 .

(below $1 \mathrm{mHz}$ ) is dominated by temperature fluctuations, which are expected to be significantly smaller when the SC is in orbit. By monitoring the fluctuations in temperature in the laboratory, the contribution could be subtracted from the OMS measurements to yield a significant increase in sensitivity. Figure 2(c) shows the expected OMS performance compared to the requirements, here expressed in terms of equivalent differential acceleration of the two test masses.

3.1.4. Reducing thruster contribution through system identification. Fluctuations in the thrust levels of the micro-Newton thrusters result in motion of the SC and hence the optical bench (which is rigidly attached to the SC). At first glance, this may appear to be a commonmode noise and as such should not appear in the differential readout, but in fact there is a residual coupling due to the spring-like couplings of the two test masses to the SC. These couplings (stiffnesses) arise from two main contributions: the local gravitational field gradient around the TM and the electrostatic stiffness due to the voltages that are applied to actuation electrodes surrounding each test mass. Since each TM and its housing are physically different and are located in different locations, these couplings will be slightly different. This difference of stiffness means that motion of the SC will couple differently to each TM and as such will 


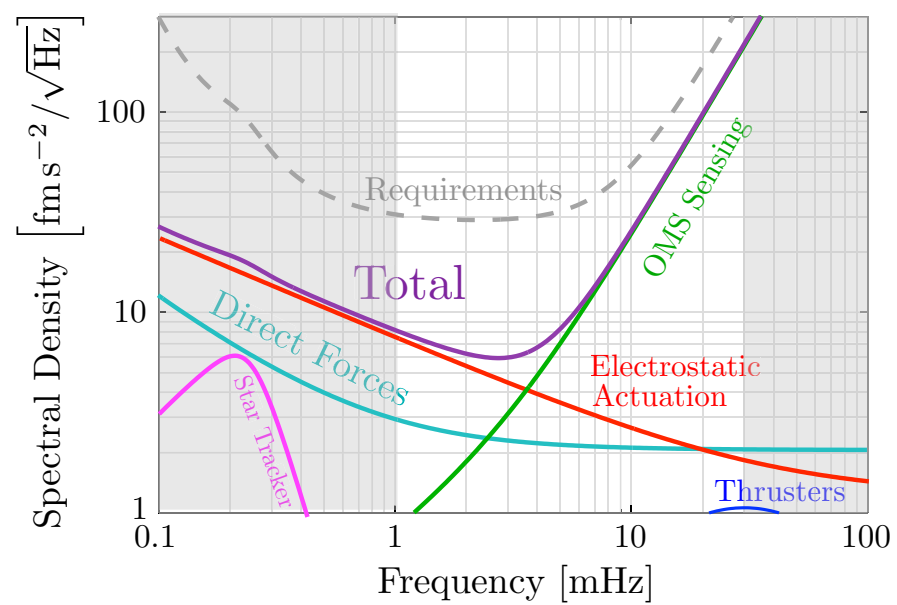

Figure 3. The differential acceleration noise budget arising from the best-case or expected performance of the individual subsystems. The acceleration noise arising from the subsystem requirements is also shown (see figure 1). Again, the grey areas are out of the measurement band for the LPF but are interesting for LISA.

appear in the differential readout. (There is also another coupling where motion of the optical bench is sensed by the differential interferometer. This is due to the geometrical construction of the optical system which results in an imperfect common-mode rejection.) This means that if we 'match' the stiffness of the two TMs, the coupling of the optical-bench motion, and hence the thruster noise, will be reduced. A series of system-identification investigations has been designed to allow determination of the stiffness terms. These can then be matched by setting appropriate voltages on the electrostatic actuators. Figure $2(d)$ shows the expected contribution of the thrusters through the coupling described above. Here, the stiffness of the two TMs is matched to a level of about 1 part in 1000 (a difference of $2 \times 10^{-9} \mathrm{~s}^{-2}$ with nominal values around $-2 \times 10^{-6} \mathrm{~s}^{-2}$ ).

\subsection{Expected performance}

In addition to the example improvements discussed in section 3.1, measurements and analyses of other aspects of the system have also revealed areas of expected improvement. For example, the contribution coming from direct forces acting on the test masses is expected to be around a factor of 10 less than the allocation. The inertial sensor hardware is designed so as to meet the more stringent requirements on force noise, such that even in the less friendly LPF environment, the performance approaches the level required for LISA. Further details can be found in [13] and [14]. Putting all of these expected improvements together, one can derive a new overall performance of the LTP-LPF system. Figure 3 shows the 'best estimate' performance breakdown derived using the arguments discussed above. One can now see that in this scenario the instrument would be limited by actuation and sensing noise. For LISA, however, this scenario is not completely pertinent, since, on LISA, there will be no $x$-axis electrostatic actuation. Although on LISA each SC contains two test masses, their sensitive $(x)$ axes are not co-linear, and as such the SC can be centred around each TM independently without the need to actuate directly on the TM. Because on the LPF the sensitive axes of the TMs are aligned, this is not possible. So a more realistic (in terms of LISA) noise breakdown 


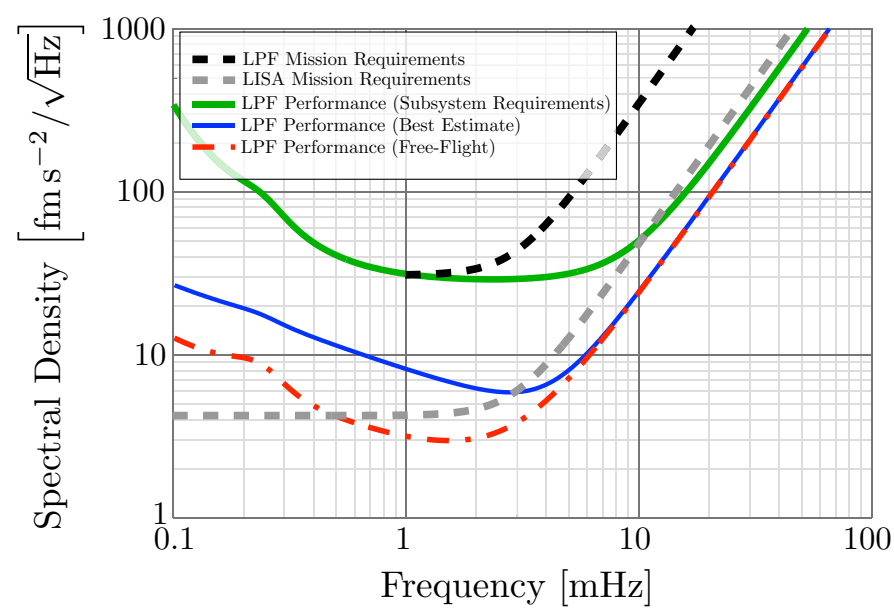

Figure 4. A comparison of different performance estimates for the LPF mission. We show the performance expected if all systems perform as per their requirements, the current best estimate performance, and the estimated performance for the free-flight experiment. The overall mission requirements for the LPF and LISA are also shown.

would not have an electrostatic actuation contribution. In fact, on the LPF an investigation is planned to probe this scenario. By switching off the electrostatic actuation, we have a system which is more representative of the LISA design. However, the actuation cannot be turned off for long; otherwise, the second test mass will drift out of its safety envelope within its housing. The experiment is designed to let TM2 drift for a fixed amount of time before recapturing it. This is then repeated many times. The data during each drift phase are then collected and analysed to estimate the differential acceleration of the TMs in the LPF measurement band. Full details of this so-called drift-mode experiment and one possible data analysis technique can be found in [15] and [16]. The expected performance during the drift mode is shown in figure 4 together with the 'best-estimate' performance and the 'subsystem requirements' performance. The figure also shows the overall mission requirements for the LPF and LISA (for differential acceleration of two test masses). One can see that during the drift mode, the expected performance of LPF improves upon the LISA goal in the LPF measurement band, and is only about a factor of 3 away at the lower end of the LISA band.

\section{Conclusions}

Gravitational wave astronomy at frequencies below $1 \mathrm{~Hz}$ will, given current technology, require a space-borne detector like LISA. By probing the frequency region around $1 \mathrm{mHz}$, LISA promises a wealth of fundamental physics and observations of systems which are unobservable in the electromagnetic spectrum. In order to ensure the success of a LISAlike mission, some key technologies and system-level principles which cannot be tested on ground will be demonstrated on the LPF satellite. In this paper, we have shown that many of the key components of the satellite are already constructed and integrated, with only a couple of outstanding items, which are needed to complete the payload (LTP) and final integration. Through analysis and testing of a large number of the flight components, the expected performance of LPF has been shown to be significantly better than the requirements. Once the remaining items are integrated into the payload, the integration of the payload and 
SC can proceed. Following that, a series of integration and system tests will lead the way to a launch, expected in mid-2014. This will be the first step to having a gravitational wave detector in space and will open the door on gravitational wave astronomy in the millihertz regime.

\section{References}

[1] Bender P et al 2000 Laser Interferometer Space Antenna: a cornerstone mission for the observation of gravitational waves Technical Report ESA-SCI(2000)11

[2] Vitale S et al 2005 Science requirements and top-level architecture definition for the LISA technology package (LTP) on board LISA Pathfinder (SMART-2) Technical Report LTPA-UTN-ScRD Iss3-Rev1

[3] Fichter W, Gath P, Vitale S and Bortoluzzi D 2005 Class. Quantum Grav. 22 S139

[4] O'Donnell J R Jr, Hsu O C, Hanson J and Hruby V 2004 Proc. 18th International Symposium on Space Flight Dynamics 548105

[5] Dolesi R et al 2003 Class. Quantum Grav. 20 S99

[6] Audley H et al 2011 Class. Quantum Grav. 28094003

[7] McNamara P et al 2011 Class. Quantum Grav. 28094001

[8] LTPDA: A MATLAB toolbox for accountable and reproducible data analysis http://www.lisa.aei-hannover. de/ltpda

[9] Hewitson M et al 2011 Class. Quantum Grav. 28094006

[10] Congedo G et al 2010 Proc. 8th Int. LISA Symp. (J. Phys.: Conf. Ser.)

[11] Nofrarias M, Röver C, Hewitson M, Monsky A, Heinzel G, Danzmann K, Ferraioli L, Hueller M and Vitale S 2010 Phys. Rev. D 82122002

[12] Ferraioli L et al 2010 Proc. 8th Int. LISA Symp. (J. Phys.: Conf. Ser.)

[13] Vitale S et al 2011 Class. Quantum Grav. 28094002

[14] Weber W J (on behalf of the LISA Pathfinder team) 2011 Proc. 46th Rencontres de Moriond

[15] Grynagier A, Fichter W and Vitale S 2009 Class. Quantum Grav. 26094007

[16] Grynagier A et al 2010 Proc. 8th Int. LISA Symp. (J. Phys.: Conf. Ser.) 